\title{
Adaptive Trajectory Control Design for Bilateral Robotic Arm with Enforced Sensorless and Acceleration based Force Control Technique
}

\author{
Nuratiqa Natrah Mansor, Muhammad Herman Jamaluddin, Ahmad Zaki Shukor \\ Faculty of Electrical Engineering, Universiti Teknikal Malaysia Melaka (UTeM), Durian Tunggal, Melaka, Malaysia
}

\begin{abstract}
This study offers an approach for tackling the issue of instability on the computed force generated on a joint of a robotic arm by improving the model of a bilateral master-slave haptic system with an adaptive technique known as Reaction Force Observer (RFOB). The purpose of recommended modelling is to correct unsought signals coming from the employed standard controller and the surroundings produced within the moving joint of the articulated robotic arm. RFOB is employed to adjust the signal interference by modifying its position response to obtain the desired final location. The investigation and observation were carried out in two separate tests to evaluate the outcomes of the recommended integration technique with the former system that only enforced Disturbance Observer (DOB). Generated feedbacks produced from the organised experiments are measured inside a simulation platform. All numerical records and signal charts illustrate the durability of the proposed method since the system integrated with acceleration-based force control is more precise and quicker.
\end{abstract}

Keywords-Force and position controller; reaction force observer; bilateral control robotic arm; sensorless; system response

\section{INTRODUCTION}

A bidirectional master-slave industrial robotic arm manipulator system is a revolutionary technology that was beyond imagination a century ago. It permits explorers and adventurers to reach the places unavailable to them. The places might be inaccessible, harmful or isolated. By the time this research is studied, there have been many applications in multiple fields, including surgical operation, exploration in the deep ocean and outer space operations, and coping with volatile or high emission activities [1]. Robots are programmable machines. It can cope in a different environment with unique features, mobile and easy to manoeuvre. Therefore, this study utilised a youBot made by German robots' manufacturer, KUKA, as the device to showcase and operate as a bidirectional haptic system.

According to prior studies conducted by researchers all around the world, old traditional approaches had technical limitations. The techniques focus on enhancing the control system itself through the use of premade and essential equipment such as a keyboard, joystick, data glove, basic manipulator connection, and the use of force sensors [2]. Common force sensors appear to have several restrictions and disadvantages for the system. The system has particular uncertainty, instability, and delays [2-4]. On an actual

\footnotetext{
Zamalah Scheme, Universiti Teknikal Malaysia Melaka (UTeM).
}

industrial robot arm, not much of the previous study uses contemporary control techniques to enhance the system feedback. It is impracticable and wasteful not to use these strategies, which have been shown to improve responses on control systems in multiple previous studies [3,5]. The combination of force control and position control into the design of bilateral robotic arms should be emphasised to discover the disparity, uniqueness, and uncommon industrial task handled by the arm manipulator with other ordinary and smaller haptic devices [6,9].

Therefore, it is a refreshing attempt to build and model a bilateral control system on industrial robots. In the past 40 years, haptic technology has evolved across engineering studies and many other research areas, including arts and design. Several researchers have studied its control system, auxiliary, communication and wearable devices, as there is a diversity of many possibilities, opening doors for incoming haptic technology [6-7]. For instance, [7,8] discovered that control action and response inside the said system might increase as far as $90 \%$ accuracy compared to the conventional approach that did not utilise any adaptive control technique. The standard system without the adjusted controller parameters needs to battle around $25 \%$ to attain the controller goal [4]. Following the deployment of DOB and RFOB, it acquired efficacy and simultaneously avoided any infallibility on the control process. With the facts, the integration of both adaptive techniques to the new design of the control system should be commissioned and emphasised. The benefits of implementing these stated observers into a system are projected to boost the feedback of bilateral haptic inside the system. It is also to eliminate all unwanted signals and disturbances that occur while operating.

The main objective of this study is to be set and incorporate a type of sensorless force control into the robotic arm simulation and observe the most acceptable parameters to arrange for the robot to operate and work optimally in a bilateral way. The outcome reported in this article is focused on employing two versions of robust control tools to increase the efficacy and discover the capability of the system to adjust its operation to obtain the optimal potential mode of operation [6-8]. The second section will delve into the robust acceleration control and the block diagram of accelerationbased force control, review the series of steps of procedures and methodologies performed on each experiment. In the following section later, all recorded data and information observed from every experiment are tallied and illustrated in 
graph version. Next, Section III will discuss the feedback of the aforementioned master-slave manipulator control system recorded from the simulation. Meanwhile, Section IV summarises the essential findings and conveys the recommendations to acknowledge the limitations and improve future work.

\section{METHODOLOGY}

\section{A. Control Design based on Disturbance Compensation}

The fundamental purpose of this project is to employ a software simulation for constructing a model of a haptic robotic arm to work in bilateral master-slave interaction. As being discussed in the introduction part, system response in normal circumstances carries noise and suffers constraints during operation. The constraints can be in the bandwidth of the filter due to the wide frequency range or internal stability. Hypothetically, there are concerns and limitations to achieving the robustness as it is generally mediocre and tough to maintain the system. One of the solutions to handle the challenge is introducing a control tool into a control system [8]. This control structure can boost system infallibility by erasing uncertainties and undesirable information within the system. The role of this inner-loop output-feedback controller is to discard outer disturbances and make up the outer-loop baseline controller resilient against the plant's uncertainties [7].

Employing force sensors in a machine or equipment brings several significant drawbacks, although it is responsible for measuring the force acting on a specific object. The conventional sensors are not exceptionally durable, expensive, and restricted capability to detect the bandwidth. This observer can be enforced into a system loop to replace the traditional instruments for estimating force measurement and remain sensorless [5]. Moreover, the developed system is suitable to perform navigation and task manipulation in a connected teleoperation setup. The dynamic characteristics of DOB can improve the restrictions and inadequate ability encountered by standard basic controllers. Compared to the other two controllers, such as Proportional and Integral controllers (PI) and Proportional Integral Derivate Controller (PID), Proportional and Derivative Controller (PD) is well matched to pair with the observer to construct a new design of closed-loop control system for the master-slave robotic arm.

Incorporating DOB in the design system can measure disturbance force, $F_{d i s}$, and give compensating current, $I_{c m p}$, achieving robust motion control in unstable plants. The proposed tool is another notable technique for measuring force-producing and estimating disturbances. Consequently, filtered data by the observer paired with a fed-back input signal will be utilised to nominalise the inner loop, satisfy causality, and adjust motion control. Therefore, it will deliver high precision readings for precise position tracking. Whereas the equation is equivalently illustrated into block diagrams as shown in Fig. 1 and Fig. 2.

Systematically, the integrated technique supports the system to predefined performance criteria and achieves firm acceleration control. Fig. 2 summarises the schematic of the acceleration-based position control block diagram.

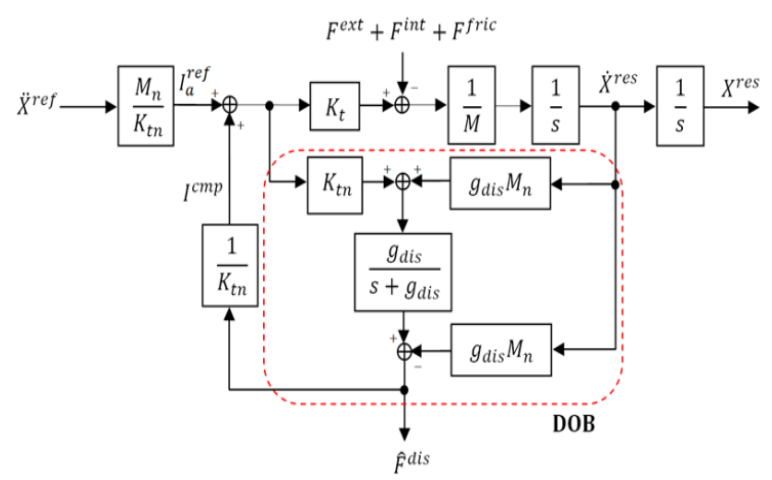

Fig. 1. A Block Diagram for a DOB based Open-loop Control System.

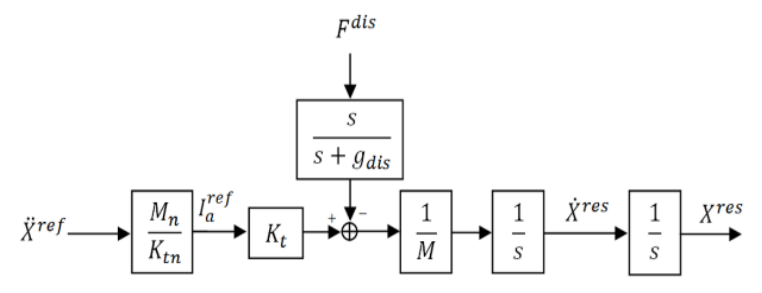

Fig. 2. Robust Acceleration Control.

The open-loop sensitivity and the co-sensitivity transfer function is derived in equation (1) below to denote uncertain and nominal plant models. The following transfer functions are the components of Fdis:

$\mathrm{F}_{\mathrm{dis}}=\mathrm{F}_{\mathrm{ext}}+\mathrm{F}_{\mathrm{int}}+\mathrm{F}_{\text {fric }}+\left(\mathrm{M}-\mathrm{M}_{\mathrm{n}}\right) \mathrm{s}^{2} \mathrm{X}_{\mathrm{res}}+\left(\mathrm{K}_{\mathrm{tn}}-\mathrm{K}_{\mathrm{t}}\right) \mathrm{I}_{\mathrm{a}}^{\mathrm{ref}}$

The force-induced consist of modelling errors of nominal mass, $M_{n}$ and nominal thrust coefficient, $K_{t n}$ in overall is represented as disturbance force, $F_{\text {dis }}$ equation. Meanwhile, Interactive force, $F_{\text {int }}$ components are the Coriolis term, centrifugal term, and also gravity term. A low pass filter (LPF) and the inverse of a nominal plant model are required to shape the DOB model. The disturbance force $F_{d i s}$ is approximated as the following equation:

$\mathrm{F}_{\text {dis }}=\frac{\mathrm{g}_{\text {dis }}}{\mathrm{s}+\mathrm{g}_{\text {dis }}} \mathrm{F}_{\text {dis }}$

The equation for position controller, $C_{p}$ in differential mode is derived from summation of both position gain, $K_{p}$ and velocity gain, $K_{v}$.

$\mathrm{C}_{\mathrm{p}}=\mathrm{K}_{\mathrm{p}}+\mathrm{sK}_{\mathrm{v}}$

Then, the corresponding position response, $x_{\text {res }}$, is expanded into:

$\mathrm{x}_{\mathrm{res}}=\frac{C_{p}}{s^{2}}\left(x_{c m d}-x_{r e s}\right)$

Where $x_{\text {res }}$ in (4) has been rearranged and translated into (5), as seen below:

$$
\begin{aligned}
& \frac{x_{r e s}}{x_{c m d}}=\frac{C_{p}}{s^{2}+C_{p}} \\
& \frac{x_{r e s}}{x_{c m d}}=\frac{s K_{v}+K_{p}}{s^{2}+s K_{v}+K_{p}} \\
& \frac{x_{r e s}}{x_{c m d}}=\frac{2 \xi \omega_{n} s+\omega_{n}^{2}}{s^{2}+2 \xi \omega_{n} s+\omega_{n}^{2}}
\end{aligned}
$$


From the above equation, the damping ratio value, $\xi$, can be tuned to 1.0 to obtain a critical damping effect, whereas natural angular frequency, $\omega_{n}$ is a substitution of $\sqrt{K_{p}}$ or $1 \frac{1}{2} K_{v}$.

\section{B. Control Design based on Reaction Force Estimation}

Aside from the original purpose of DOB, it can be employed with RFOB to estimate the reaction force. Past studies have shown that the RFOB can estimate a wider band and excellent range of situations (Mansor et al., 2017). Consequently, force sensors are replaceable to be employed in bilateral manipulator's systems. The component of estimation also requires the identification of friction force $F_{\text {fric }}$ and the interaction force $F_{\text {int }}$. Furthermore, the design of RFOB is competent to estimate the exterior force given out by the disturbance of the components in the type of acting force or force response [4-5]. To describe the process of this control loop technique and arrangement within the loop system, outlined block diagrams is illustrated in Fig. 3 and Fig. 4, respectively.

The cut-off frequency, $g$ for RFOB, is similar to the DOB. Thus, the computed external force $\hat{F}_{\text {ext }}$ is equate as follows:

$\hat{F}_{\text {ext }}=\frac{g_{\text {dis }}}{s+g_{\text {dis }}} F_{\text {ext }}$

As the value for force controller, $C_{f}$ is associated with the force gain, $K_{f}$, hence the estimated force response, $F_{\text {res }}$ is described as follows:

$\hat{F}_{\text {res }}=\frac{C_{f} z_{c} g_{\text {dis }}}{s^{2}\left(s+g_{\text {dis }}\right)}\left(F_{c m d}-\hat{F}_{\text {res }}\right)$

Which can be transformed into (8):

$\frac{\hat{F}_{\text {res }}}{F_{c m d}}=\frac{1}{\frac{s^{2}\left(s+g_{d i s}\right)}{C_{f} Z_{c} g_{d i s}}+1}$

\section{Design of Bilateral Master-Slave System with Adaptive Control Technique}

Fig. 5 displays the whole close-loop bilateral control system following the employment of both control loop techniques into the system. The observers' data will merge and feed back into the input signal for every passing process. The generated feedback will correct any internal modelling error or interruption and emerge into one input.

There are two modes in the bilateral control system, which are Differential Mode, $\ddot{x}_{d i f}$ and Common Mode, $\ddot{x}_{\text {com }}$. The first mode is the product of position controller, $C_{p}$ with the difference between the position of the master-slave system, and the latter is the product of force controller, $C_{f}$ with the differences between the forces computed in master-slave. The equation for both modes follows the equation (9) and (10) below.

$\ddot{x}_{\text {dif }}^{r e f}=C_{p}(s)\left(x_{s}^{r e s}-x_{m}^{r e s}\right)$

$\ddot{x}_{c o m}^{r e f}=C_{f}(s)\left(f_{s}^{e x t}-f_{m}^{e x t}\right)$

\section{Procedures for Work Simulation}

This study's analysis and experiment are carried out through a simulation platform inside a robotic simulation software called Virtual Robotic Experimentation Platform
(VREP). The software has a built-in KUKA youBot in its library and is ready to be integrated with various programmable tasks and coding languages. Furthermore, simulation scenes, models, and object characteristics are simple to manage as it has formed a plethora of choices and functionalities. The first task to be considered to prove whether the proposed system is ideal must follow the law of action and reaction in bilateral communication. After the system works according to the law, the experiment will be carried out to analyse the system with adaptive techniques. All starting values for each parameter and setting are presumed to be related to the real-time experiment. To construct the bilateral way of communication inside the KUKA youBot, the controllers, input and output arrangement are shown in Fig. 6.

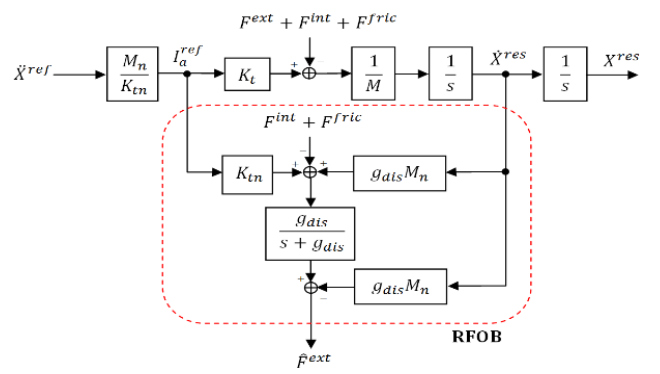

Fig. 3. A Block Diagram for RFOB based on an Open-Loop Control System.

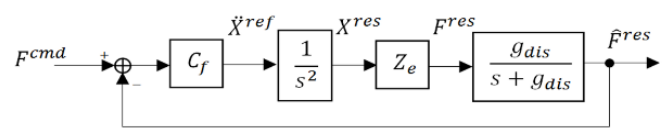

Fig. 4. Acceleration based Force Control System Block Diagram.

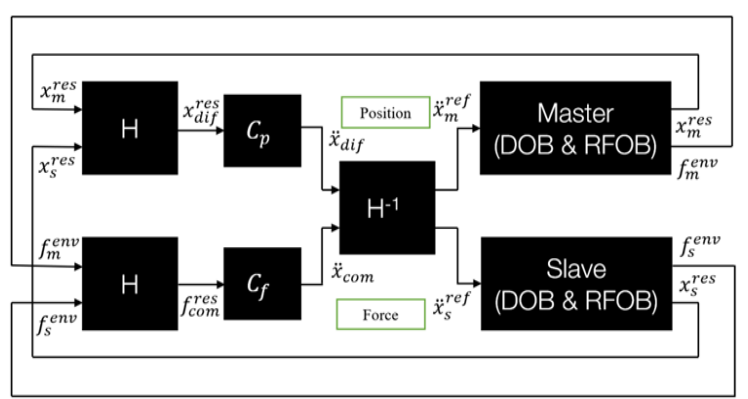

Fig. 5. A Simplified Block Diagram of Master-Slave Bilateral Control System with Proposed Tools.

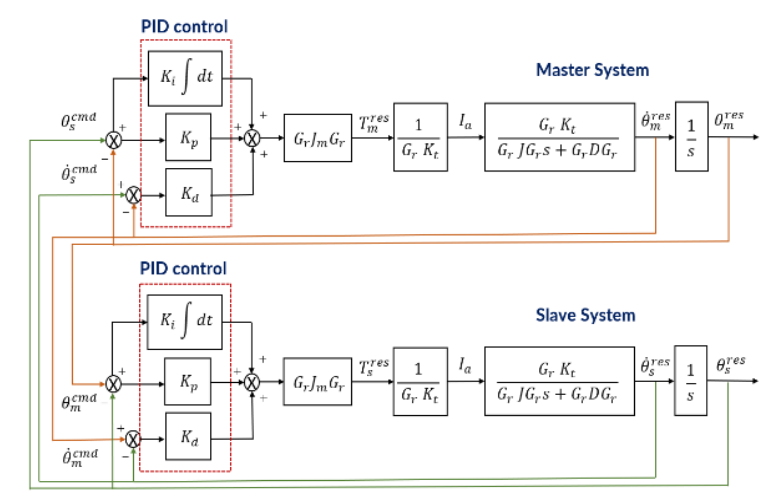

Fig. 6. General Block Diagram for a Bilateral Robotic Arm with Two Separate Subsystems. 
The remote Application Programming Interface (API) function will be used to communicate between both robots with the programmed environment. To simplify the study work, only reading in a single joint are monitored even though the robot has 5 degrees of freedom (DOF) and multiple operatable joints, as indicated in Fig. 7 below. This joint on the waist part is labelled as 'Joint0' (located at the first joint on the lower robot component).

The reason to focus on a single link of the manipulator is to reduce the complexity of managing the trajectory control and operating successive joints and degrees of freedom during tests. The environment and setup for all experiments are modelled in VREP. To illustrate the simulation processes, the steps are outlined in Fig. 8 and shown in the following Fig. 9a and Fig. $9 \mathrm{~b}$.

In summary, this section explained the methodology to carry the experiment, general block diagrams for every proposed design of bilateral control system and introduced observer, modelling equations and pictorial illustration of the robot operating in simulated software. Detail procedures and control setup have been described to explain every test that has been carried out.

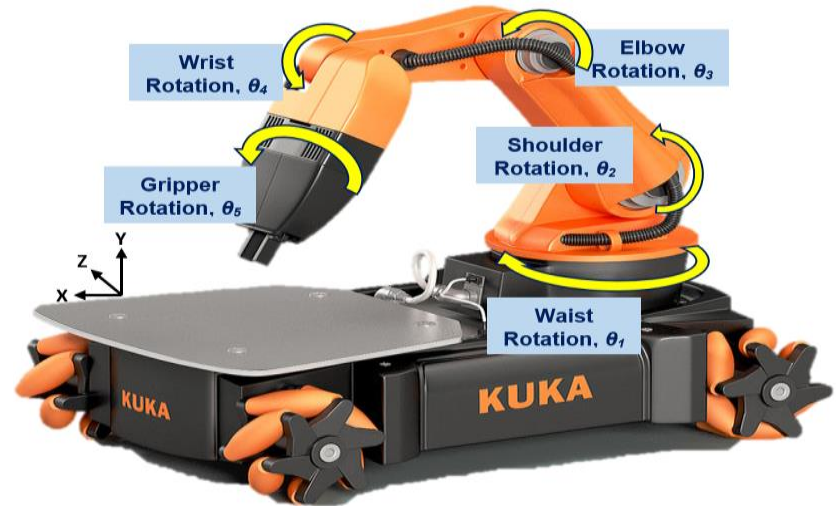

Fig. 7. Visualisation for Five Degrees of Freedom (DOF) of the YouBot Arm.
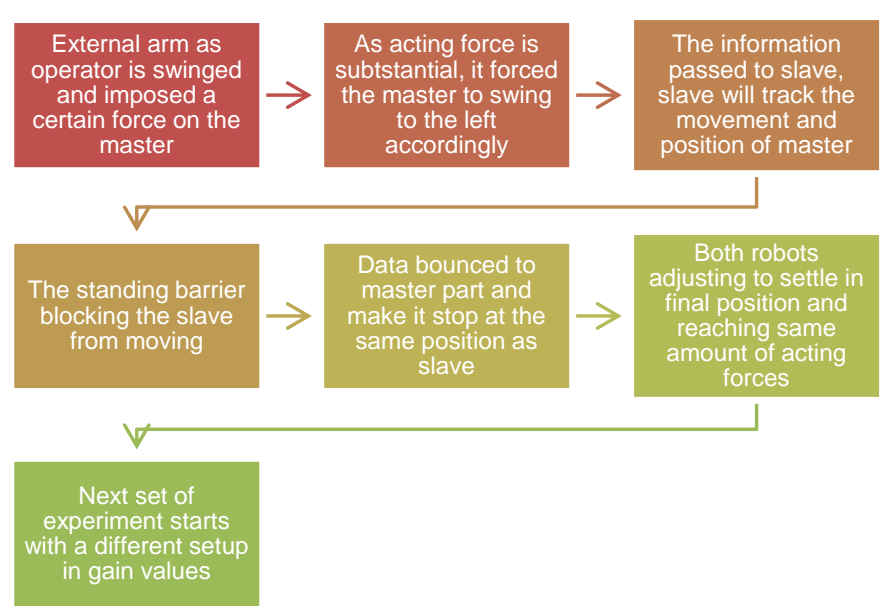

Fig. 8. Procedure Undergoes by the Robotic Manipulator.

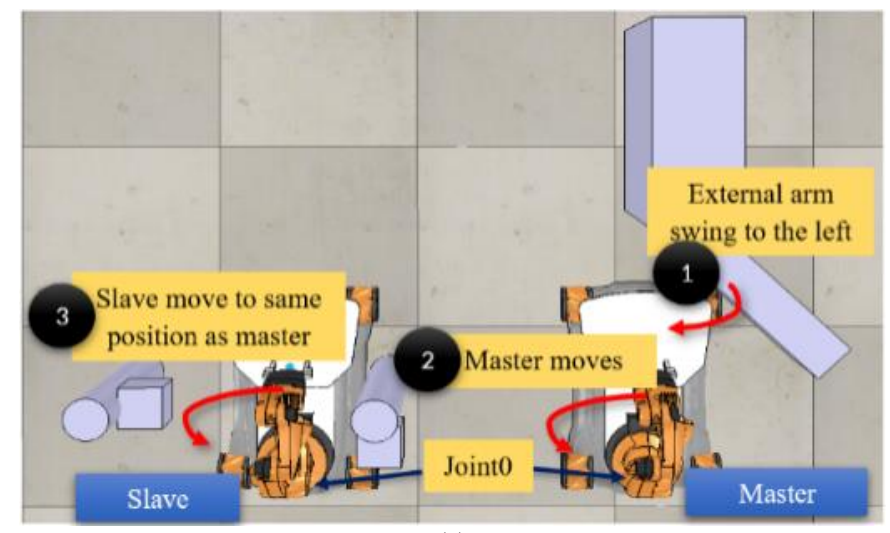

(a)

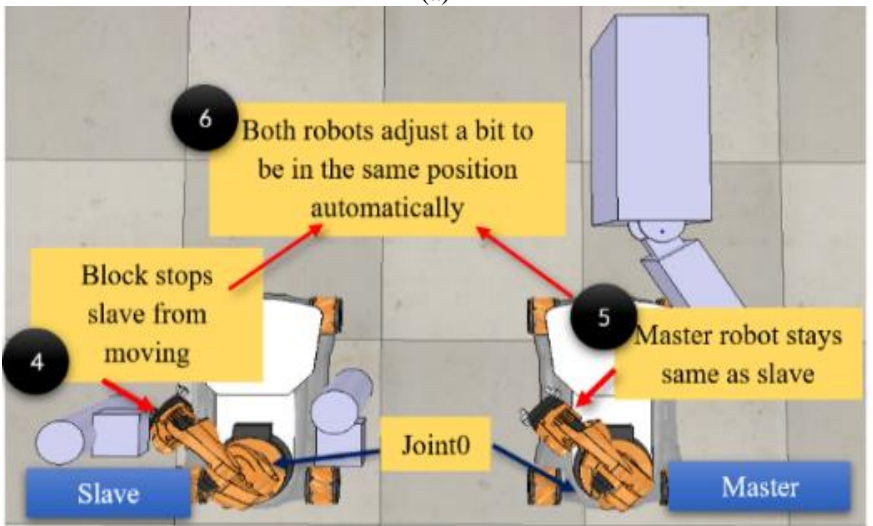

(b)

Fig. 9. (a) and (b). Overall view and Operation Steps for the Bilateral Control System.

\section{RESUlTS AND ANALYSIS}

This section discusses the proposed system's outcome, and output feedback gathered after running separate tests. All findings from experiments have been acquired and assembled into table form and constructed into graph form to observe the disparity between all versions of control systems. Each experiment has been conducted according to the parameters and variable setlist. For every refreshed assessment set, all steps are repeating for three times to compute the mean values before being illustrated in the graph version. These independent variables of $K_{p}$ and $K_{d}$ values are presented in Table I below. For RFOB, the priority is to validate the Differential Mode Law of the bilateral master-slave telerobotic arm manipulator system. The test is to verify that the system abiding by the law of subtraction between master and slave for position reading is equal to zero. The test also demonstrates that the integration of two types of different observers is capable of enhancing the system response and lowering the noise value within the internal system. The selected gain values are based on the experimental validation approach. Numerous trials have been performed on an extensive range of variables to determine appropriate lowest and highest values that work compatibly with the proposed system. 
TABLE I. IDENTIFIED SET OF CONTROLLER GAIN VALUES

\begin{tabular}{|l|l|l|}
\hline$\omega_{n}$ & $K_{p}\left(\omega_{n}\right)^{2}$ & $K_{d}\left(2 \omega_{n}\right)$ \\
\hline 1 & 1 & 2 \\
\hline 2 & 4 & 4 \\
\hline 5 & 25 & 10 \\
\hline 10 & 100 & 20 \\
\hline 20 & 400 & 40 \\
\hline 50 & 2500 & 100 \\
\hline 100 & 10000 & 200 \\
\hline 200 & 40000 & 400 \\
\hline 500 & 250000 & 1000 \\
\hline
\end{tabular}

\section{A. Evaluation on Force Control}

The first analysis is performed with RFOB is paired to the inner loop output feedback of the working system. The proposed control loop is implemented to monitor the force reaction happening in the system. The recorded force reading generated on both subsystems is displayed in Fig. 10 to Fig. 18, respectively.

Entire graphs from Fig. 10 to Fig. 18 shows the feedback in forces for master and slave youBot in a different set of settings, corresponding to every value of $\omega_{n}$ as listed in Table I. There are two types of forces produced in every graph. The first generated formed in blue colour signifies the torque reading yield from the master; meanwhile, the second line in orange represents the torque induced from the joint at slave robot. It should be noted that the maximum torque for Joint0 for both subsystems is set at $8 \mathrm{~N}$.

Referring to graphs in Fig. 10 to Fig. 14, when the force in the master began to grow into $8 \mathrm{Nm}$ after being pushed by the external manipulator, the reading value in the slave varied and generated a series of gradual increments in force reading. At this point, the slave did not move and remained in its original position at $0^{\circ}$. This is because the gain values of $K_{p}$ and $K_{d}$ are too small and insufficient to increase the controller's sensitivity in the proposed bilateral control system. Although the force reading in the master climbed up for a certain period after being pushed, the feedback and data circulated from the master subsystem were considered ignored by the slave. This is because the slave cannot read the exact information passed through its subsystem. Force generated on the said joint is collectively unstable and fluctuating.

Nevertheless, as exposed in Fig. 10 to Fig. 12, force reading on both systems is restored to zero after the external manipulator returned to its early position, stopping it from pushing the master youBot arm ahead. There are some instabilities traced in force reading of master and slave as shown in Fig. 13 and Fig. 14 after the value of $K_{p}$ and $K_{d}$ is increased up to $K_{p}=100, K_{d}=20$ and $K_{p}=400, K_{d}=40$. At this point, force reading became unstable and wavering, evidently referred to the current scenario in the simulation workspace. Both robots attempt to identify each other's final pose when the slave robot quakes after being in contact with the obstruction block.

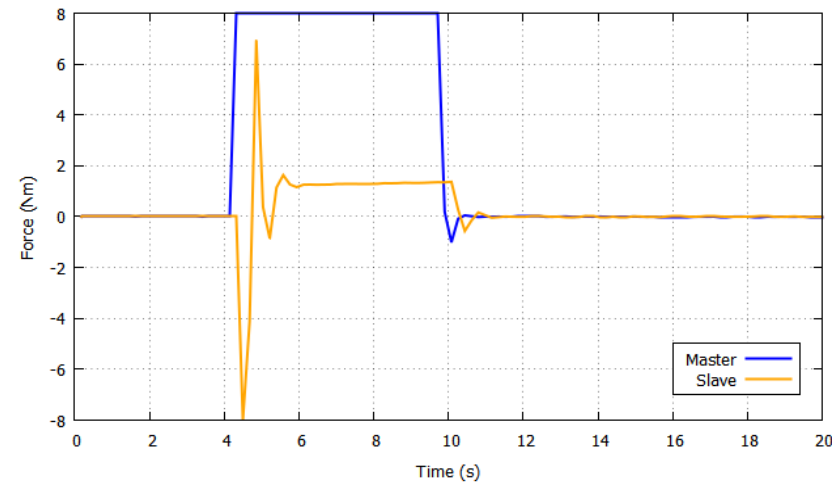

Fig. 10. Generated Force on both Subsystems across Period for $\omega_{\mathrm{n}}=1$.

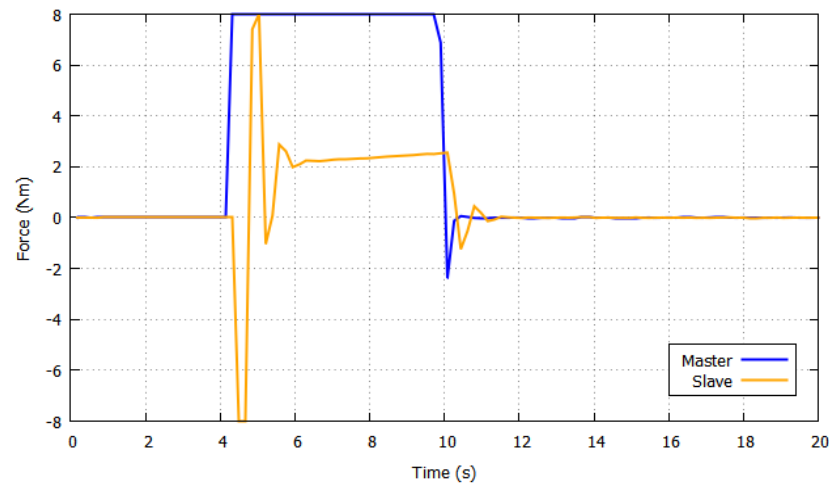

Fig. 11. Generated Force on both Subsystems across Period for $\omega_{n}=2$.

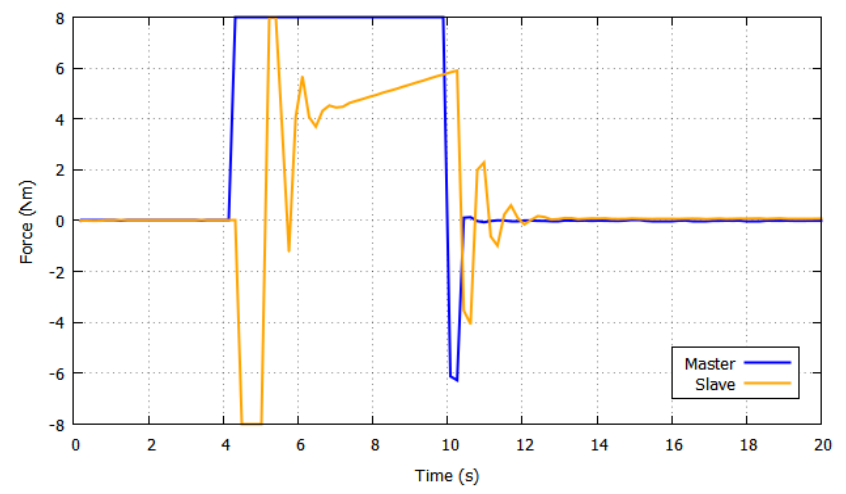

Fig. 12. Generated Force on both Subsystems across Period for $\omega_{n}=5$.

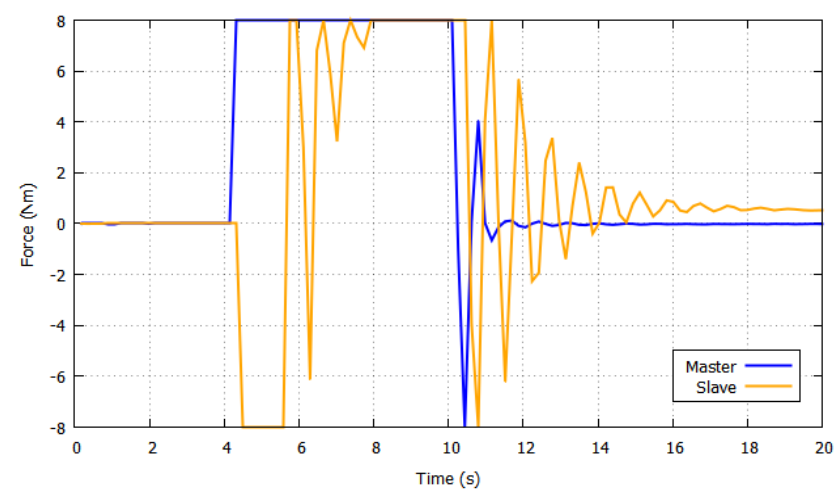

Fig. 13. Generated Force on both Subsystems across Period for $\omega_{n}=10$. 


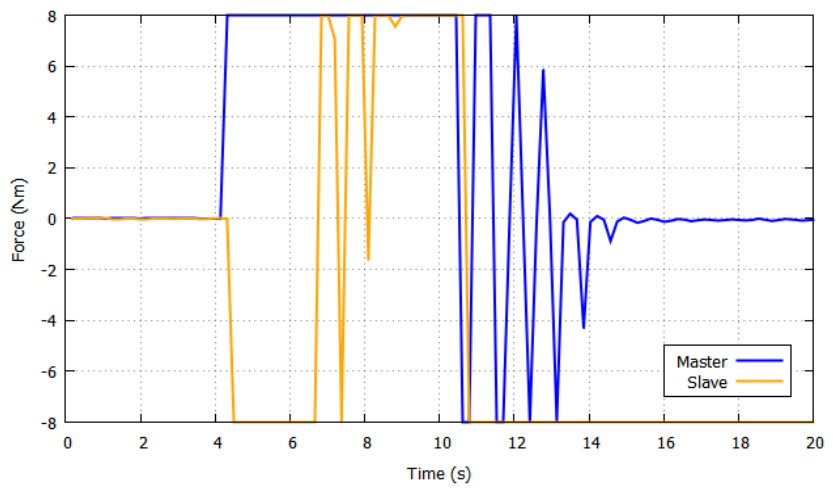

Fig. 14. Generated Force on both Subsystems across Period for $\omega_{n}=20$.

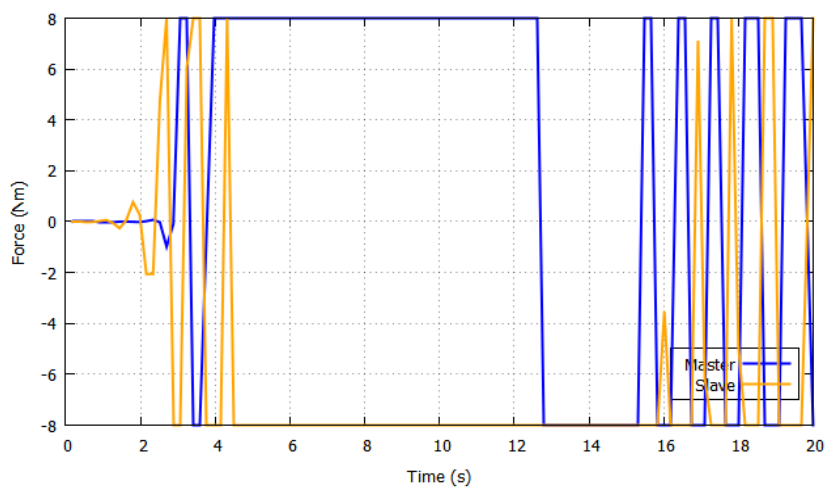

Fig. 15. Generated Force on both Subsystems across Period for $\omega_{\mathrm{n}}=50$.

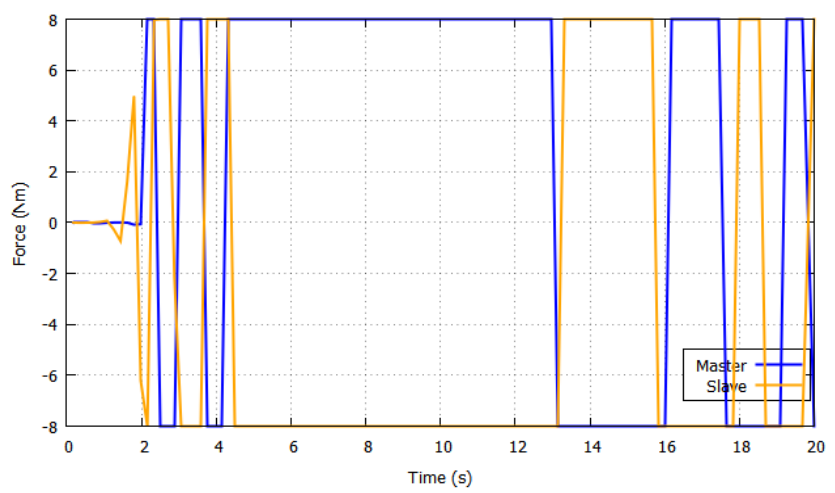

Fig. 16. Generated Force on both Subsystems across Period for $\omega_{\mathrm{n}}=100$.

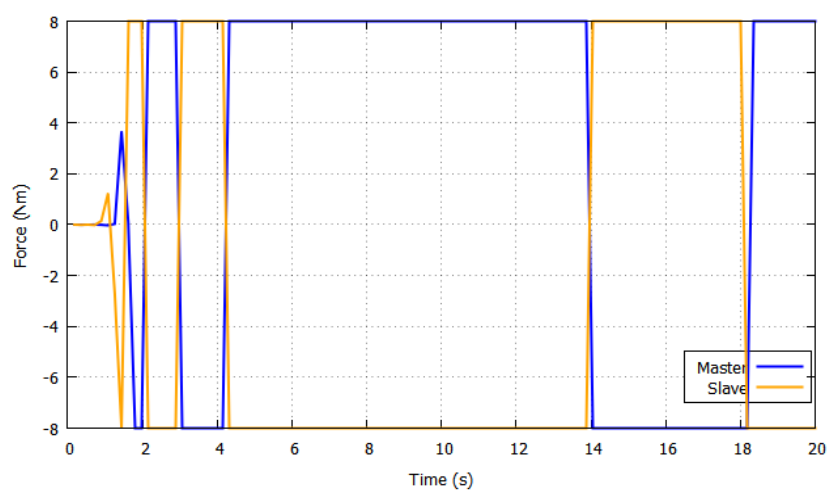

Fig. 17. Generated Force on both Subsystems across Period for $\omega_{n}=200$.

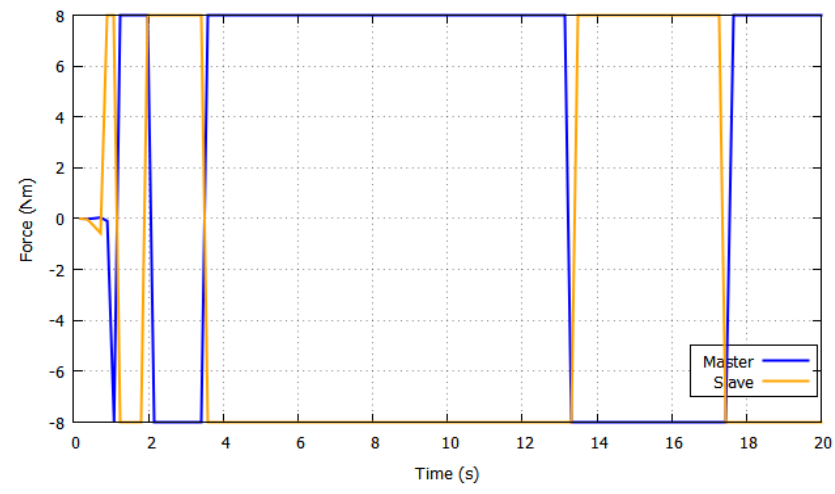

Fig. 18. Generated Force on both Subsystems across Period for $\omega_{\mathrm{n}}=500$.

Whereas in Fig. 15 to Fig. 18, force reading at slave's joint shows a finer trend of the desired output and corresponds to the reading in master's joint compared to the prior. The gain value for each parameter has been more extensive. At $\mathrm{t}>3.5 \mathrm{~s}$, the external arm began to press on the surface of the master youBot. After receiving information from the partner identified as master youBot, the slave youBot will detect the same applied force and proceed ahead. When the block stopped the slave, it instantly applied a counterforce response and attempted to move forward. The shape of the graph can describe this condition in Fig. 15 to Fig. 18. Graphs revealed that the master youBot arm used its maximum torque of $+8 \mathrm{~N}$ to go further. However, the slave youBot arm attempted to withstand greater force coming from the block, resulting in $8 \mathrm{~N}$ of torque reading in the experiment. The greater the gain values of $K_{p}$ and $K_{d}$ were thrust to the system, the greater the connection between the magnitudes of the input signal and the magnitude of the output signal in a steady state. In short, force reading in blue showed that the master youBot arm delivered a maximum torque valued at $+8 \mathrm{~N}$ to move. In contrast, the slave youBot arm tried to counter the enormous force from the block, which resulting $-8 \mathrm{~N}$ of opposing torque value in the experiment. After $\mathrm{t}>12 \mathrm{~s}$, the external manipulator returned to its original position and ceased to exert $8 \mathrm{~N}$ of torque for pushing the master youBot arm. At this moment, there is no outside force acting on the master youBot to propel it forward. Nonetheless, the force reading can be traced on both sides of master and slave as these two are swinging back and forth before settling on their initial position, which is at $0^{\circ}$. Taking the force reading from the graph and simulation of the system, the robots oscillated for a short time as they strive to settle and eradicate the value of the disturbance before returning to the position in proceeding.

\section{B. Evaluation of Position Control}

The second experiment aimed to recognise position control for both single links of the youBot arm (at Joint0) when RFOB is implemented to enhance and operate the system. Thus, all graphs from Fig. 19 to Fig. 27 display the position readings of Joint 0 for each master and slave robotic arm.

Every graph from Fig. 19 to Fig. 27 above depicted the position feedback of the single joint in master and slave youBot in a separate set of parameters ranging from $\omega_{n}=1$ to $\omega_{n}=500$, associated to a list of $\omega n$ determined in Table I. The blue line indicates the reference angle, also known as step 
input, while the green line in the graphs indicates the joint position of the master, and lastly, the red line signifies the joint position of a slave.

Initially, both Joint0's master and slave manipulator positions are set at $0^{\circ}$. Referring to graphs in Fig. 19 to Fig. 20, the reading for the position of Joint0 in master youBot reached its peak time at around $\mathrm{t}>9.5 \mathrm{~s}$, and the situation was maintained until $\mathrm{t}=20 \mathrm{~s}$. The position of slave youBot touched the highest at $15^{\circ}$ in Fig. $21,5^{\circ}$ in Fig. 20 and $2^{\circ}$ in Fig. 19. In comparison, the position of a slave has minimally increased after $\mathrm{t}>5 \mathrm{~s}$, although it did not have much difference compared to the original position. The position of the master reached its peak time values around $70^{\circ}$ at $9.5 \mathrm{~s}$, as portrayed in Fig. 19 to Fig. 21, while $10 s$ for Fig. 22 to Fig. 23 and $78^{\circ}$ at $13.5 \mathrm{~s}$ in Fig. 24.

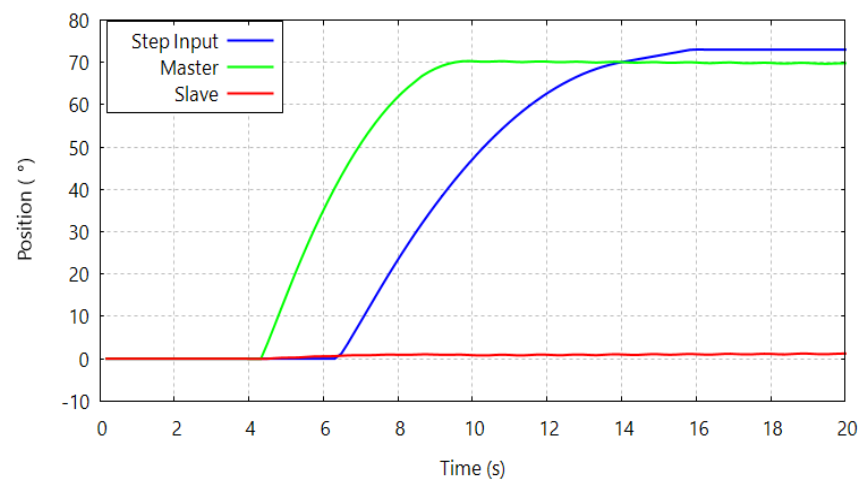

Fig. 19. Position Tracked on both Subsystems across the Period for $\omega_{n}=1$.

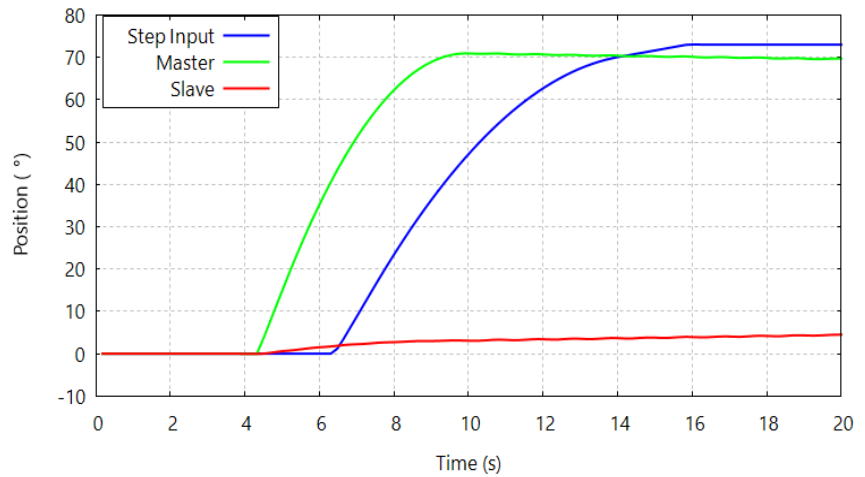

Fig. 20. Position Tracked on both Subsystems across the Period for $\omega n=2$.

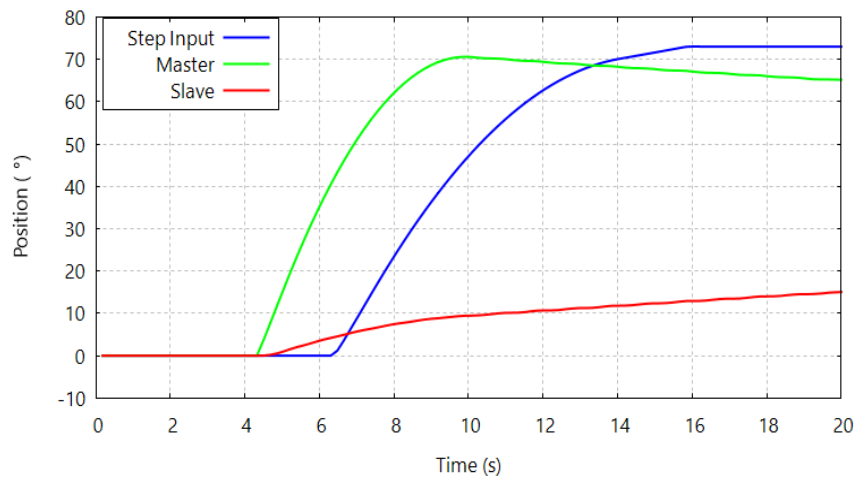

Fig. 21. Position Tracked on both Subsystems across the Period for $\omega n=5$.

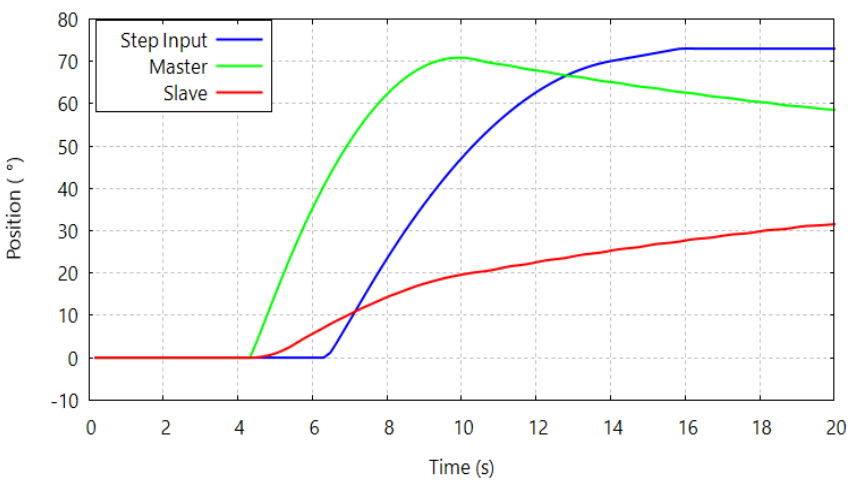

Fig. 22. Position Tracked on both Subsystems across the Period for $\omega n=10$.

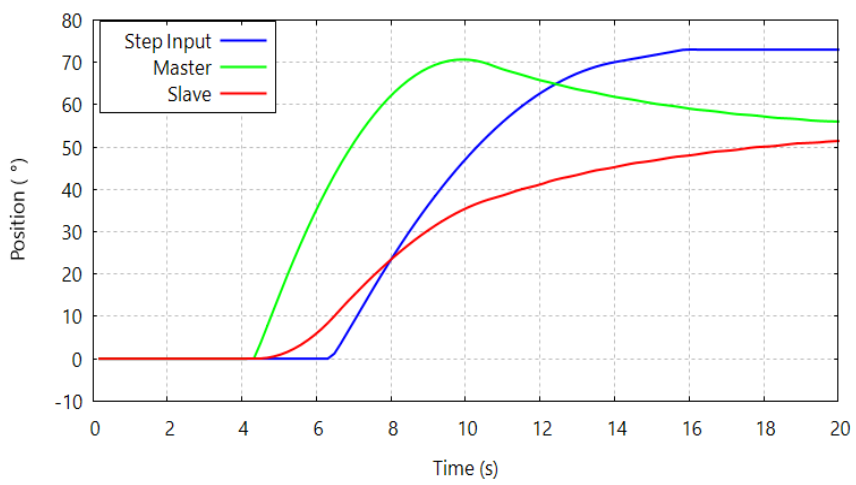

Fig. 23. Position Tracked on both Subsystems across the Period for $\omega n=20$.

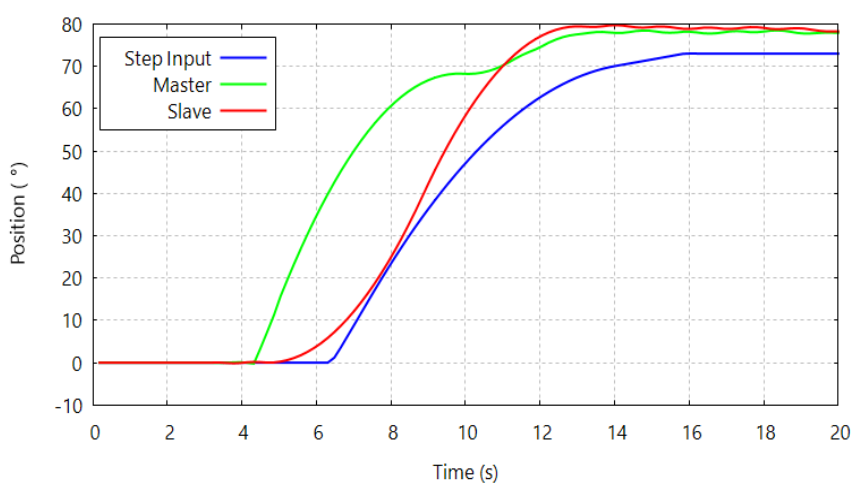

Fig. 24. Position Tracked on both Subsystems across the Period for $\omega n=50$.

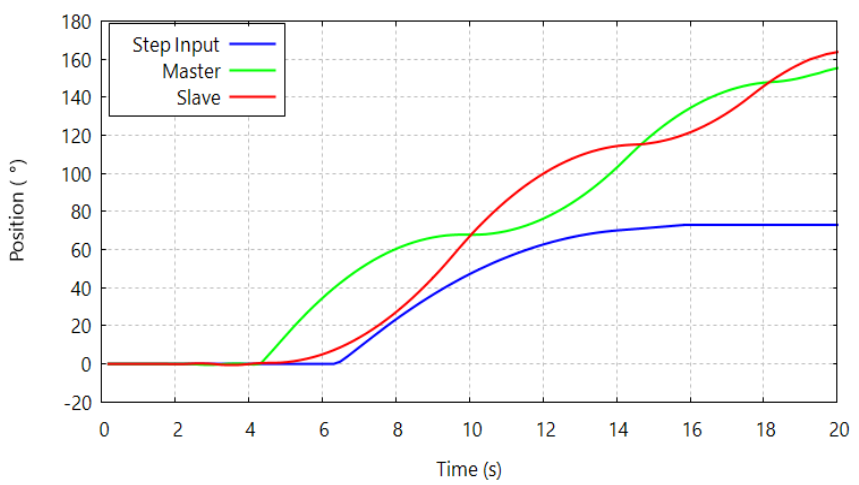

Fig. 25. Position Tracked on both Subsystems across the Period for $\omega n=100$. 


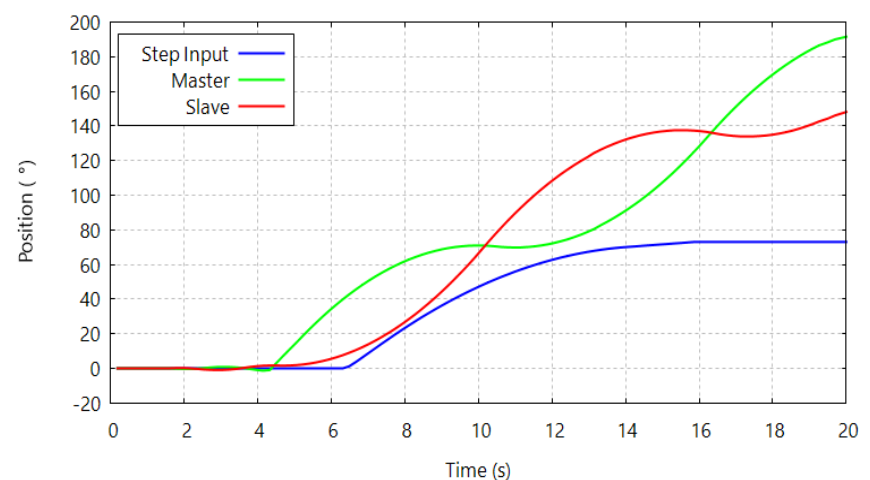

Fig. 26. Position Tracked on both Subsystems across the Period for $\omega n=200$.

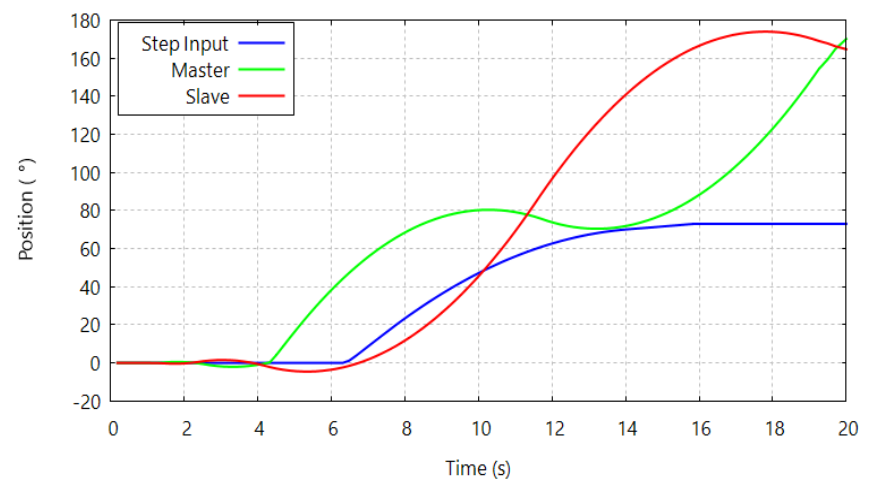

Fig. 27. Position Tracked on both Subsystems across the Period for $\omega n=500$.

While for Fig. 25 to Fig. 27, both master and slave alternately swing while gradually increasing on its position. The reading from the graphs kept going up because the master arm received an external force that made it move forward. However, the position of the slave according to Fig. 19 until Fig. 21 changed in minimal value compared to the master arm robot because the gain value is not adequate, having neither effects of the percentage of overshoot nor settling time. Referring to Fig. 24 and Fig. 27, when the value of gain is increased, the angle of position in the slave robot started to have changed, moved further and stretched at the top position, recorded at $80^{\circ}$ for stable condition and $178^{\circ}$ for unstable condition. On the other hand, the reading for the master's position began to drop, similar to the slave's position reading, as shown in Fig. 24. Following the graphs in Fig. 25 until Fig. 27 , it can be demonstrated that slave youBot was trying to move further to track the position of master and master concurrently trying to catch the latest post of the slave.

Nevertheless, delays are noticed during the communication process of the two subsystems, thus making the position reading fluctuate until the end of the experiment. The position value of master and slave surpassed the reference angle when the value of $\omega_{n}$ is 50 until the value of $\omega_{n}$ was set at 500 . Graph reading in Fig. 24 also presented that the differences in the value of error between the position of master and slave with reference angle are the lowest compared to others which are noted at $-6^{\circ}$ to $-7^{\circ}$, and the most significant error is observed from the graph in Fig. 19 with the value of $-70^{\circ}$. Based on the output reading, the percentage of accuracy for each design control is computed and tabulated into a table form. Table II below shows that control systems using RFOB have achieved greater accuracy compared to other systems, while Table III organised the transient response of various frequencies.

TABLE II. ACCURACY RECORDED IN EVERY DESIGNED SySTEM

\begin{tabular}{|l|l|l|l|}
\hline$\omega_{n}$ & $K_{p}\left(\omega_{n}\right)^{2}$ & $K_{d}\left(2 \omega_{n}\right)$ & Accuracy $(\%)$ \\
\hline 1 & 1 & 2 & 1.78 \\
\hline 2 & 4 & 4 & 6.49 \\
\hline 5 & 25 & 10 & 23.09 \\
\hline 10 & 100 & 20 & 53.79 \\
\hline 20 & 400 & 40 & 91.82 \\
\hline 50 & 2500 & 100 & 98.94 \\
\hline 100 & 10000 & 200 & 99.54 \\
\hline 200 & 40000 & 400 & 92.66 \\
\hline 500 & 250000 & 1000 & 95.31 \\
\hline
\end{tabular}

TABLE III. COMPARISON OF TRANSIENT RESPONSE FOR VARY FREQUENCIES

\begin{tabular}{|l|l|l|l|l|l|l|}
\hline \multirow{2}{*}{$\omega_{n}$} & \multicolumn{2}{|l|}{ Peak Position $\left(^{\circ}\right)$} & \multicolumn{2}{l|}{ Peak Time $(\boldsymbol{s})$} & \multicolumn{2}{l|}{ Delay $(\boldsymbol{s})$} \\
\cline { 2 - 7 } & Master & Slave & Master & Slave & Master & Slave \\
\hline 1 & 70 & 2 & 9.5 & 20.0 & 4.5 & 6.0 \\
\hline 2 & 70 & 5 & 9.5 & 20.0 & 4.5 & 5.0 \\
\hline 5 & 70 & 15 & 9.5 & 20.0 & 4.5 & 5.0 \\
\hline 10 & 70 & 32 & 10.0 & 20.0 & 4.5 & 5.0 \\
\hline 20 & 70 & 52 & 10.0 & 20.0 & 4.5 & 5.0 \\
\hline 50 & 80 & 80 & 13.5 & 13.5 & 4.5 & 5.0 \\
\hline 100 & 158 & 162 & 20.0 & 20.0 & 4.5 & 5.0 \\
\hline 200 & 190 & 170 & 20.0 & 20.0 & 4.5 & 5.0 \\
\hline 500 & 170 & 178 & 20.0 & 17.5 & 4.5 & 7.0 \\
\hline
\end{tabular}

From nine designed systems, six of them achieved more than $90 \%$ of accuracy. The system achieved the most accuracy with $\omega_{n}=50$, steadily at $99.78 \%$, followed by $\omega_{n}=500$ and $\omega_{n}$ $=20$, with each of them reaching more than $95 \%$ accuracy. Meanwhile, the system with the most negligible accuracy for differential mode law is recorded at $3.56 \%$ when the value of $\omega_{n}=1$. Therefore, the best design of the proposed system to acquire the most satisfactory position control is $\omega_{n}=500$. This is because master and slave robots attained the same final position after $15 \mathrm{~s}$, had a minor steady-state error, and achieved critical damping. For common mode law, the ideal design for the controller is when $\omega_{n}=50$. As prove, the summation of torque reading observed at both joint of master and slave system revealed to be equal to zero and experienced more minor disturbance as seen in Fig. 19.

\section{DISCUSSION AND CONCLUSION}

To sum up, the outstanding response derived from all analyses for both experiments, the results are outlined in Table IV accordingly. The most satisfactory outcome is highlighted as the best parameter for each mode law. 
TABLE V. RESPONSE IN BOTH LAW OF BilAteral CONTROL SyStem

\begin{tabular}{|l|l|l|}
\hline & Common Mode & Differential Mode \\
\hline Compatible & $K_{p}=250000$ and $K_{d}=1000$ & $K_{p}=2500$ and $K_{d}=100$ \\
\hline Incompatible & $K_{p}=1$ and $K_{d}=2$ & $K_{p}=250000$ and $K_{d}=1000$ \\
\hline $\begin{array}{l}\text { Delay and } \\
\text { overshoot }\end{array}$ & & $-0.5 \mathrm{~s}$, from $11 \mathrm{~s}$ to $15 \mathrm{~s}$ \\
\hline Accuracy & $\begin{array}{l}\text { Summation of forces } \\
\text { between master and the } \\
\text { slave is zero }\end{array}$ & $\begin{array}{l}\text { Position difference between } \\
\text { master and slave is almost } \\
\text { zero }\end{array}$ \\
\hline Fotal & $\begin{array}{l}\text { Fewer vibrations, more } \\
\text { stable }\end{array}$ & \\
\hline
\end{tabular}

According to the above summary in Table II, $K_{p}=2500$ and $K_{d}=100$ are considered as the best gain for the integrated system. Once RFOB is employed into the system, six systems achieved greater than $90 \%$ accuracy, compared to only five systems that reached more than $90 \%$ accuracy without the RFOB. Above all, the form of noise produced at Joint0 in the second experiment is much more refined than in the first experiment. This proves RFOB has advantages in improving the system stability and eliminating periodic oscillation. Furthermore, the recommended technique is intended to remove unwanted signals such as Coriolis forces, $F_{c}$, viscous damping friction, and gravity forces produced internally, especially on the motor located at a specific link. To achieve an accurate estimation of forces to be delivered to the operator, the dynamical effects within the force signal must be adjusted.

The RFOB experiment required around $11 \mathrm{~s}$ to $15 \mathrm{~s}$ to attain an overshoot in terms of the time delay. In RFOB configuration, the settling time generated by slave youBot is considerably better and sharper. Furthermore, the amplitude of the curve formed is relatively constant and persistent until the target joint reaches its final position. Nevertheless, the system is underdamped for a moment before it progressively climbs to reach overshoots and peak times. For the record, underdamped is a condition in which the system oscillates slower at a low-frequency rate and takes longer to get a steady-state. This situation occurred whenever the value of the PD controller increased. As a result, the stability of the control system may be derived to be conditional, based on the value of gain and threshold. In overall, the proposed designs can reach stability in a certain level of gain levels but can deteriorate when the gain value is unsuitable.

The main objective to validate a master-slave control system with DOB and RFOB implementation abiding by the law of bilateral control system has been successfully confirmed. The stated target is justified by running several sets of parameters and collecting the output response for the analysis. The suggested technique has met the capacity in enhancing the whole system performance. This study also demonstrates that a control system can be sensorless to measure dimension when active reacting forces are in contact with the system. The proposed technique is also applicable to multiple applications of industrial robots aside from position and force tracking. Using the observers to replace old-style force sensors on a device or equipment increased the system reaction and improved internal uncertainty across the system.
In conclusion, few analyses are performed to determine every single type of common controller response, rankings in the percentage of accuracy, overshoot, settling time and delay. The best kind of controller was chosen based on its performance in all three studies. This conclusion is backed by the fact that both robots oscillated at a reduced angle for a time in a control system without applying the DOB approach before they stabilised and came down to remain in a single spot. Whereas the idea for introducing RFOB into the system will remove the unwanted disturbances and errors that arrive before being feed at the DOB loop. Different experiments with diverse parameters have been conducted to oversee the system's latency after applying DOB and RFOB as part of the control loop technique. This work also assesses the system reaction for each suggested design of the bilateral system with a varied set of controllers. Indirectly, the primary purposes of this study were successfully attained.

Several analyses were performed to determine each controller's performance, standings in the accuracy, timedelay, and settling time. The best controller was chosen based on its performance in all three studies. This conclusion is supported by the fact that both robots oscillated at a smaller angle for a while in a control system without employing the DOB technique before they stabilised and came down to remain in a single place. Whereas the method to add RFOB into the system will subtract the uncertainties coming on the input of DOB. To observe the potential and advantage of applying DOB and RFOB onto the system response, separated tests with different parameters have been performed to examine and evaluate the system reaction for each proposed controller design. Indirectly, the second and third objectives of this study were successfully achieved.

Several proposals may be offered and studied in the near future research to enhance the system and make it more resilient. First, video and visual input and recording might be implemented by putting a high-speed camera at the tip of the robot arm as an added sensor for tracking. The extracted data from raw images and videos will undergo image processing technique into a type of visual force to suppress the signal error and remove the unwanted noise [10]. Besides, the relevant data gained from the pictorial records will be utilised to increase the coordination of the system's trajectory [11]. The visual data feeding into the reaction force estimation loop system will be used for the soft navigation system. Assuredly, the research should be undertaken on actual hardware and real interaction to assess the response and stability in a real-time experiment.

Additionally, this project can be one of the working mobile robots dispatched to risky or work in remote regions due to its versatility. Aside from that, a specific experiment is recommended to execute by employing image processing technology on Linux based operating system with a robot operating system (ROS) connected to the entire robotic arm. The information processed from visual data can be fed into the system for more outstanding object tracking and positioning accuracy. The limitations of executing the current technique on a simulator and virtual platform would lower the capacity of the produced feedback and overall system performance. This control procedure is likely to be more responsive in 
actual ROS communications. It is easy to track any unaligned output tracking or missteps while executing the robot's task. Not just that, implementing a real-time based system for subsequent study can boost the time responsiveness on the machine and actively doing force and position tracking.

\section{ACKNOWLEDGMENT}

The authors would like to acknowledge the funding supplied by Universiti Teknikal Malaysia Melaka (UTeM) under the Scheme of Zamalah to perform the study. The authors would also like to convey our credits to the Robotics and Industrial Automation research group within the Faculty of Electrical Engineering.

\section{REFERENCES}

[1] N. Hiroshi, O. Kiyoshi, Y. Yuki, K. Naoki, M. Toshimasa and T. Akifumi, "Force Sensorless Fine Force Control Based on Notch-Type Friction-Free Disturbance Observers," IEEJ J. Ind. Appl., vol. 7, 2018, pp. 117-126.

[2] Al. Kolsanov, S. Chaplygin, S. Rovnov and A. Ivaschenko, "Augmented Reality Application for Hand Motor Skills Rehabilitation,” Int. J. Adv. Comput. Sci. Appl. (IJACSA), vol. 11, no. 4, 2020.

[3] G. Liming, Y. Jianjun \& Q. Yingjie, "Torque control based direct teaching for industrial robots considering temperature-load effects on joint friction," Ind. Rob., vol. 46 no. 5, 2019, pp. 699-710.
[4] U. Kodai, S. Tomoki, T. Kenta, S. Sho and T. Toshiaki, "Reaction Force Estimation of Electro-hydrostatic Actuator Using Reaction Force Observer,” IEEJ J. Ind. Appl., vol. 7, 2018, pp. 250-258.

[5] J. Seul and L. Joon, "Similarity Analysis Between a Nonmodel-Based Disturbance Observer and a Time-Delayed Controller for Robot Manipulators in Cartesian Space," IEEE Access, vol. 9, 2021, pp. 122299-122307.

[6] S. Choi and K. J. Kuchenbecker, "Vibrotactile Display: Perception, Technology, and Applications," Proc. -2013 IEEE, vol. 101, no. 9, 2013, pp. 2093-2104.

[7] E. Sariyildiz, and K. Ohnishi, “A Comparison Study for Force Sensor and Reaction Force Observer-based Robust Force Control Systems", Proc. -2014 IEEE 23rd Int. Conf. Ind. Electron., 2014, pp. 1156-1161.

[8] E. Sariyildiz, S. Hangai, T. Uzunovic and T. Nozaki, "Discrete-Time Analysis and Synthesis of Disturbance Observer-Based Robust Force Control Systems," IEEE Access, vol. 9, 2021, pp. 148911-148924.

[9] N. N. Mansor, M. H. Jamaluddin and A. Z. Shukor, "Concept and application of virtual reality haptic technology: A review", J. Theor. Appl. Inf. Technol., vol. 95, no. 14, 2017, pp. 3320-3336.

[10] A. Protsenko, "The Development of a Fault Detection and Identification System for Executive Units of Manipulators Using Technical Vision," Int. Multi-Conf. Ind. Eng. Mod. Technol. FarEastCon, 2020, pp. 1-5.

[11] Z. I. Bell, P. Deptula, H. Chen, E. A. Doucette and W. E. Dixon, "Velocity and Path Reconstruction of a Moving Object Using a Moving Camera," Proc. Am. Control Conf., 2018, pp. 5256-5261. 